\title{
Filigrane
}

Écoutes psychanalytiques

\section{Introduction. Le travail de la consultation psychanalytique. Entre cadre psychique et cadre institué}

\section{Isabelle Lasvergnas}

Volume 27, numéro 2, 2018

La consultation psychanalytique aujourd'hui, entre héritages et remaniements

URI : https://id.erudit.org/iderudit/1055745ar

DOI : https://doi.org/10.7202/1055745ar

Aller au sommaire du numéro

Éditeur(s)

Revue Santé mentale au Québec

ISSN

1192-1412 (imprimé)

1911-4656 (numérique)

Découvrir la revue

Citer ce document

Lasvergnas, I. (2018). Introduction. Le travail de la consultation

psychanalytique. Entre cadre psychique et cadre institué. Filigrane, 27(2), 7-16.

https://doi.org/10.7202/1055745ar 


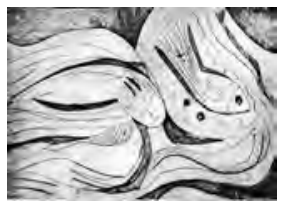

\section{Introduction. Le travail de la consultation psychanalytique. Entre cadre psychique et cadre institué}

Isabelle Lasvergnas

1 arler de la consultation psychanalytique aujourd'hui, c'est s'inscrire dans les mouvements de l'approfondissement métapsychologique qui a accompagné depuis les années 1960 l'extension de la psychanalyse très audelà du champ de la névrose. C'est prendre la pleine mesure de l'importance mutative produite dans les dispositifs thérapeutiques et la théorie de la cure par l'expérience croissante de la rencontre clinique avec des patients relevant des pathologies «limites», des troubles narcissiques-identitaires et de certaines formes de psychose.

Est-il encore besoin d'évoquer Freud qui dès 1926 pressentait que «l'utilisation de l'analyse pour la thérapeutique des névroses n'est qu'une de ses applications », ajoutant: "peut-être l'avenir montrera-t-il que ce n'est pas la plus importante» (Freud, 1926).

Un auteur comme Jacques André ${ }^{1}$ a déjà soutenu que le patient étatlimite est le patient-type pour la psychanalyse, et de la psychanalyse. Cette affirmation, qui laisse entendre que c'est la détresse psychique de ces patients, beaucoup plus encore que la douleur névrotique, qui constitue l'objet théorique central, déplace le curseur de l'interrogation très en deçà de la conflictualité odipienne et ses multiples théâtres de l'interdit pour attribuer la prééminence aux impasses dans la symbolisation, et de ce qui, dans le symptôme, ne parvient pas pour le patient à intégrer le champ de la représentation (V. Cornalba, infra; Josée Leclerc, infra).

La problématique des expériences psychiques primaires non métabolisées qui occupe désormais très largement le devant de la scène analytique s'exprime par un recentrement très affirmé sur le modèle freudien de la seconde topique: ce dont André Green à titre emblématique témoigne tout 
au long de son œuvre dans l'attention portée aux constellations psychiques caractérisées par un défaut de contenance d'affects invasifs, une non-différenciation entre le dedans et le dehors, et la prédominance d'un pulsionnel de décharge, faisant des sujets atteints de ces signes symptomatologiques des esclaves de la quantité ${ }^{2}$. Sans omettre les ajouts fondamentaux dans la lignée des interrogations successives de Ferenczi, Bion, Winnicott, Anzieu, sur la fonction psychique contenante, digestive et interprétative de la psyché maternelle à l'endroit des éprouvés bruts hors langagiers de son nourrisson.

C'est dans ce contexte théorique et culturel de la clinique contemporaine que s'était inscrit le dialogue inter-centres de traitements psychanalytiques tenu à l'occasion d'un colloque à Montréal à l'automne 2016, et que prolonge la présente publication ${ }^{3}$.

Le tableau qui y fut dressé a rendu compte des approches de la consultation dans plusieurs organismes aux traditions plus ou moins anciennes et plus ou moins établies: en France, les éminents Centre de consultation et de traitements psychanalytique (CCTP) Jean-Favreau rattaché à la Société psychanalytique de Paris, Centre Évelyne et Jean Kestemberg relevant depuis sa fondation de l'Association de santé mentale du $13^{\mathrm{e}}$ arrondissement à Paris (ASM13), Centre Smirnoff relié à l'Hôpital Sainte-Anne et le BAPU Pascal ${ }^{4}$; ainsi qu'au Québec, constitué beaucoup plus récemment, Groupe psychanalytique du Mont-Royal (GPMR).

La plupart de ces centres furent largement associés aux débats d'écoles qui ont caractérisé la pensée psychanalytique française depuis près de six décennies. Certains se sont affirmés comme le lieu d'avancées novatrices sur la question des premières rencontres et leurs dispositifs, ainsi que sur les modalités d'une prise en charge conjointe, psychiatrie/accompagnement psychanalytique.

Tous les centres susnommés, sans exception, furent fondés dans la volonté d'élargir socialement l'accès à une écoute psychanalytique pour des patients dont la précarité interne et la souffrance psychique se trouvent redoublées dans la plupart des cas par une situation de grande vulnérabilité économique. Une précision, cependant: si la préoccupation de promouvoir une équité sociale dans l'accès à la psychanalyse pour les moins nantis ${ }^{5} s^{\text {'ins- }}$ crit dans le sillage des souhaits de Freud, Abraham et Etingon au Congrès de Budapest en 1918, elle ne suffit pas en soi pour faire d'un organisme offrant des traitements gratuits ou semi-gratuits ${ }^{6}$ une instance de recherche clinique. La grande portée d'enseignement et la fécondité de la contribution des centres de traitements dont les témoignages sont rassemblés dans cette 
publication, tiennent à une mise de l'avant de positions théoriques princeps qui ont présidé à leur création, conférant à chacun d'eux une identité bien particulière qui s'est traduite de surcroit dans des philosophies organisationnelles très spécifiques. Elle tient également à leurs réflexions sur une pratique située aux limites de la psychanalyse classique, sur leur capacité de réélaboration des positions originelles qui avaient présidé au fondement de leurs missions, et à l'inventivité de psychanalystes souvent «sans divan» mettant à l'épreuve de multiples jeux du cadre.

Selon les particularités et l'histoire propres à chacun des organismes, l'accent principal sera mis sur des aspects spécifiques et constitutifs de leurs cadres de traitements. Ainsi, dans le cas du Centre Kestemberg, on systématisera une approche psychanalytique pilote du traitement de la psychose (Gibeault, infra; Kapsambelis, 2012, 2017). Dans les BAPU fondés en France au lendemain de la Seconde Guerre mondiale et destinés aux étudiants, il s'agira de leur offrir un soutien pluriel, dont une écoute analytique, et de se centrer sur des formes de détresse plus généralement propres à leur classe d'âge (Ballif, infra). Dans le cas du GPMR - et dans celui de la Clinique psychanalytique de Montréal (CPM) - où est mis en place un dispositif d'accueil plurisubjectif pour les premières rencontres avec un patient ${ }^{7}$, on visera à systématiser la portée théorico-clinique de ce dispositif original (Lasvergnas, 2018a, et infra). Tandis que sera réaffirmé au Centre Smirnoff, dans les conditions empiriques de la mise en place des traitements, le principe d'un caractère «privatissime» de la cure, fidèle en cela à l'esprit de Victor Smirnoff fondateur du Centre dont il deviendra l'éponyme (Homer-Koffi, infra) : un principe qui met en sourdine la potentialité de Nebenmensch associé de l'institution dans la relation duelle patient-thérapeute, et sa fonction de matrice de contact (Cornalba, infra). Ou, plus exactement, un principe qui juge contradictoire à l'éthique analytique l'accent mis dans l'organisation de certains dispositifs de la prise en charge des cures sur l'espace transférentiel que peut virtuellement occuper l'entité organisme-clinique pour le patient. Un positionnement de principe qui à cet égard, marque une prise de distance par rapport aux propositions d'Évelyne Kestemberg sur la portée d'inscription psychisante de l'institution et celle du personnage-tiers chez le patient. Ou encore à celles de Jean-Luc Donnet pour qui une des contributions théoriques les plus claires de «l'exigence transgressive» du tiers institutionnel est d'ouvrir un espace psychique élargi, l'ouverture de l'espace intra-analytique sur l'espace inter-analytique constitué par le double cadre institutionnel manifestant «la pertinence et l'envers (de ce) double cadre 
qui conjoint/disjoint le cadre de la séance et celui de l'institution» (Donnet, 2009; voir aussi Kaswin, infra $)^{8}$.

On dit que la psychanalyse est en crise. Sans aucun doute est-ce le cas du modèle idéal-type divan-fauteuil avec rencontres de trois à quatre fois par semaine, dont l'indication thérapeutique se restreint aujourd'hui de manière tout à fait notable dans le contexte des transformations culturelles profondes des dernières décennies et du changement majeur des modalités de demandes de soins adressées au psychanalyste.

Mais le dispositif de la cure-type fut-il jamais généralisable, et son ascèse de neutralité relationnelle requise de la part du thérapeute fut-elle jamais adéquate pour l'ensemble des problématiques psychopathologiques? Ferenczi très tôt avait entrevu plusieurs inadéquations des présupposés théorico-techniques de ce cadre pour les patients souffrant de traumas psychiques graves.

Tout autant qu'un certain archétype de la psychanalyse se trouve aujourd'hui ébranlé dans les représentations populaires, le sont aussi la plupart des grands garants méta-sociaux organisateurs des sociétés occidentales et leurs résonances intrapsychiques plus ou moins directes. Néanmoins, on sait que la puissance de bouleversement et de subversion de conventions et de normes communes que produisent les périodes historiques de crise a pour effet de lever le voile sur un ensemble d'implicites considérés comme un allant de soi laissé jusqu'alors en l'état d'un non questionné/non questionnable, et d'engendrer par extension un renouvellement de la pensée.

De la puissance des mouvements culturels de fond qui retentissent sur sa pratique, le psychanalyste n'ignore rien, pas plus qu'il ne peut s'en abstraire dans la nécessité qui est devenue la sienne de remettre en cause les fondements de certains de ses propres habitus professionnels normés et d'un surmoi transmis - et, corollairement, en complexifiant son approche soignante, de redéfinir son inscription référentielle dans la Cité.

Être aujourd'hui, psychanalytiquement parlant, présent au temps présent, c'est se faire le témoin des répercussions sur la scène inconsciente des profondes mutations socioculturelles en cours. C'est se pencher sur les transformations d'une Kulturarbeit dont les nouvelles coutumes en émergence dans un être-ensemble atteignent les manières de penser et d'agir individuelles et collectives, ainsi que les modalités d'endiguement ou de non-endiguement des manifestations d'une désintrication pulsionnelle. C'est porter la plus grande attention aux bouleversements des codes de la communication intersubjective qui contaminent l'usage des mots de la langue, et qui 
souvent en altèrent, sinon en détruisent, la profondeur sémantique. À cela, ajoutons la modification profonde du rapport au temps social, et celle tout aussi marquée des rapports socio-symboliques entre les sexes et des codes de «l'échange amoureux», avec leurs prolongements sur ce qui se déroule dans une scène d'apparente réalité de corps à corps sexués. Sans compter chez de très nombreux patients le choc de la rencontre entre des univers de civilisations étrangères les unes aux autres, ou encore les déflagrations intrapsychiques de la nature des «névroses actuelles» chez des populations migrantes ou des individus soumis à des traumas de masse (Ballif, infra).

Ces multiples expressions d'un «malêtre» (Kaës, 2012) qui, sans relever véritablement de la psychose, échappent largement au modèle référentiel de la névrose, imposent à la psychanalyse un renouvellement de son approche thérapeutique et des modalités de l'interprétation (Gauthier, infra). Ce qui empiriquement parlant impliquera de la part du clinicien une concentration sur des difficultés qui ne sont pas seulement d'ordre clinico-technique, mais reliées aussi à des apories notionnelles. Par exemple, celles auxquelles le confronte la fréquentation de patients que plusieurs auteurs (Pinard, infra) qualifieront de «difficiles» pour tenter de cerner, en dehors des classifications nosographiques du type DSM IV et V, l'hétéronomie de certaines dynamiques «des limites» par rapport aux modèles topiques freudiens plus établis, plus familiers, et éventuellement - c'est là un piège possible de l'écoute contre-transférentielle - plus syntones au Moi de l'analyste.

Dans la notion, centrale dans son œuvre, de «site analytique», Jean-Luc Donnet a mis l'emphase sur le caractère très condensé de la représentation de La psychanalyse, en premier lieu chez les cliniciens. En tant que catalogue géologico-spatialisé de l'ensemble des moyens participant de l'offre de la cure, le site rassemble dans des rapports mutuels d'emboitements modèles théoriques, dispositifs cliniques, maniement de la cure et institutions psychanalytiques.

Autrement dit, lorsqu'un analyste instaure un cadre de traitement particulier avec un patient, cette décision n'est pas seulement un acte technique hautement spécialisé. Il participe d'un transfert sur le site au sens générique du terme, et à travers celui-ci de la transmission intergénérationnelle de l'acte fondateur de la discipline et de ses développements subséquents amplifiés par les filiations d'écoles et leurs effets doctrinaires. Mais si acte fondateur et corpus conceptuels constituent bien un point de ralliement de la communauté analytique et une part de l'identité de ses membres, il peut advenir que ce point de ralliement devienne un point de fermeture. J.-L. 
Donnet dira: risque de positions «intégristes» dans des représentations plus ou moins figées du cadre clinique et dans des impératifs surmoïques de sa mise en ouvre. C'est une même dénonciation tout aussi ferme qu'exprimait Didier Anzieu lorsqu'il écrivait en 1976:

L’usure gagne plus ou moins vite tout système pédagogique [...] thérapeutique ou mystique [...]. La psychanalyse est tombée malade de son succès. Il arrive que la doctrine devienne un dogme, un art, une routine, la cure un rituel, et la formation psychanalytique une carrière [...]. Le conservatisme l'emporte chez beaucoup de psychanalystes sur l'innovation. Narcisse supplée à Edipe. La tragédie cède le pas à la stratégie [...]. La psychanalyse en est arrivée là, à ce moment-ci de son histoire, à ce point. (1976, p. 252)

S'inscrivant à son tour dans la même perspective critique de ce qu'il nomme "position idéologique» (2016), René Kaës a écrit à propos des nouveaux défis qui se posent à la psychanalyse et des polémiques qu'ils suscitent en termes de méthode, de cadres de travail et de remaniement métapsychologique, qu'ils font problème "au triple sens que la langue grecque confère à ce mot: problema est à la fois un obstacle à surmonter, un sujet de controverse et une protection pour avancer davantage en terrain inconnu ou hostile.» (Kaës, 2015, p. VI).

Le paradoxe et la double figure d'un obstacle dont on souligne le caractère de protection, pointent la difficulté des efforts d'une mise en pensée oscillant entre résistance au changement, et désir de dépassement d'un fixisme de l'héritage. Ils désignent l'écueil de répétitions dont les formes et les contenus ne peuvent être identifiés que dans le retour en après-coup sur la rencontre clinique, mais également sur celui, exigeant, d'une réécriture de l'histoire des institutions analytiques et au cœur de celles-ci, du refoulement d'alliances narcissiques dans les mouvements d'une transmission inter-transférentielle et intergénérationnelle - dont l'alliance Freud-Fliess avait constitué le premier préliminaire (Kaës, 2014).

Les très importants développements métapsychologiques sur le groupe et le noyau groupal du Moi des dernières décennies m'ont personnellement amenée à établir une distinction entre instituer, au sens actif de fonder, et l'institué (participe passé), au sens de ce qui est déposé chez le sujet dans les multiples éléments d'une transmission internalisée et destinée à être reproduite. Instituer et fonder impliquent une filiation et un transport d'héritage porteurs parfois de ruptures ou de remaniements plus ou moins radicaux. 
Tandis que l'institué dans sa dimension d'une «passéité» du Moi sous-entend un ensemble de traces de l'ordre de mécanismes identificatoires fusionnants et d'alliances inconscientes. Ces deux processus, l'actif d'une pensée tendue vers des représentations-but, et le passif d'inscriptions refoulées, infiltrent les dynamiques institutionnelles. En ce sens, ce n'est pas le moindre mérite de l'expérience du travail dans un centre de consultation et dans l'effet de grossissement d'optique que produit l'échange inter-analytique que de voir portées à la lumière des répétitions instituées - au sens précis où je l'entends de fragments identificatoires inconscients - dont l'enracinement subjectif et/ou groupal d'une conviction surmoïque se répercute dans les idéologies de la mise en œuvre des cures. Néanmoins, les résultantes doctrinales explicites ou au contraire plus obscures de ce que je nomme l'institué chez le sujet ou dans le groupe ne sont pas parfaitement monolithiques: Martin Gauthier (infra) rappelle que l'identification s'enracine «dans le terreau de l'ambivalence», pavant en cela la voie à des possibilités d'ouvertures et de relectures subjectivées.

«Consultation psychanalytique» est un terme polysémique, et il convient de s'attarder à la pluralité de ses significations. En premier lieu, dans la signification la plus étroite du mot, «consultation» désigne un acte professionnel qui est inscrit dans une temporalité objectivement datable et circonscrite, à savoir celle des rencontres préliminaires entre un thérapeute et un patient. Rencontres que J.-L. Donnet (1997, 2005, 2013), dans la double fonction qu'il leur reconnaît, qualifie précisément de «consultatives», fonction opératoire objectivante pour le patient en termes d'esquisse provisoire de diagnostic structurel et d'indication de traitement dans un spectre large de cadres thérapeutiques ${ }^{9}$; et, côté analyste, fonction auto-consultative de son désir d'engagement avec le patient, fonction d'auto-observation ou d'interlocution interne et en séance dont J.-F. Chiantaretto (infra) a proposé une esquisse métapsychologique, en particulier avec sa notion de «témoin interne».

La consultation est ici à entendre littéralement comme une première rencontre entre deux psychés dans les tours et détours des valences affectives de la communication inconsciente que contient sémantiquement le mot anglais «encounter». "Rencontre» désigne à proprement parler une première rencontre dans la convocation de celle-ci d'une temporalité psychique particulière chez le patient, inscrite dans un imaginaire apte à solliciter le travail de l'après-coup. C'est du moins la potentialité d'une ré-écriture de son histoire interne figée que cherche à repérer le thérapeute dans 
ces moments d'antichambre d'un traitement à venir chez la personne qui s'adresse à lui pour la première fois (Baldacci, 2013).

Mais, par-delà la temporalité de l'événement d'une rencontre intersubjective qui prend place dans le temps social circonscrit des premiers rendezvous, et qui sollicite chez le patient la mobilisation d'un complexe mnésique tissé de fantasmes refoulés, de souvenirs-écrans, d'angoisses, de projections, etc. qui lui sont singuliers, la langue française accorde à ce même terme de «consultation», du strict point de vue de la temporalité, une signification plus diffuse, en référence au déroulement d'un processus qui n'est jamais une fois pour toutes installé, et toujours asymptotique. La consultation psychanalytique réfère alors au travail qui se déploie dans le transfert et le contre-transfert, et dans l'accompagnement théorétique qui sous-tend le cheminement de l'écoute singulière qui est prêtée à un patient «à nul autre pareil» (Dufresne, infra).

Ainsi, l'expression «consultation psychanalytique», dans sa double acception du terme français, c'est-à-dire dans son sens étroit de ponctuation psychique chez le patient des premières rencontres avec un analyste et leurs dispositifs, et dans son sens élargi d'un travail thérapeutique progrédient, peut figurer comme la métonymie des paramètres de l'écoute clinique. Je parlerai donc du travail de la consultation, au sens de travail psychique, pour désigner un espace processuel d'élaboration et un cadre contenant; et, dans les termes méthodologiques, de problèmes à résoudre, pour dépeindre la recherche d'un approfondissement des «voies de la thérapie psychanalytique» (Freud, 1919) dans la signification générique du terme, et dans l'unicité de chaque thérapie particulière. Sans rien banaliser des principes de la méthode et de l'éthique analytiques, ni se fondre dans le vaste marché des psychothérapies en tous genres que propose notre époque.

«La consultation psychanalytique aujourd'hui, entre héritages et remaniements », titre-thème de la présente monographie, retraverse cette pluralité de significations. Étayé sur l'exemplarité de plusieurs vignettes cliniques, ce recueil vise à baliser dans une transversalité théorique et dans l'enrichissement réflexif que permet le dialogue un des champs interrogatifs les plus actuels et les plus importants dans la discipline. Il témoigne des interrogations que pose à un analyste œuvrant dans un centre de traitements analytiques la spécificité de sa pratique dans un tel contexte, ainsi que sa richesse, et le renouvellement d'éclairage qu'il peut apporter, par opposition ou comparaison, à sa pratique privée. 


\section{Notes}

1. Cité de mémoire.

2. Pour faire nôtre l'expression de Michel de M'Uzan.

3. Le colloque sur le thème de La consultation psychanalytique aujourd'hui. Temps premiers de la rencontre et dispositifs thérapeutiques, tenu à Montréal le 29 octobre 2016, constituait un des éléments d'un projet de recherche interuniversitaire franco-québécois (Université de Paris 13, Université de Paris Sorbonne-Cité, UQÀM). Le projet intitulé «Dispositifs thérapeutiques et institutions de soins: enjeux cliniques contemporains de la consultation thérapeutique/Therapeutic settings and care-providing institutions: contemporary clinical issues in therapeutic consultation", avait bénéficié de subventions partielles de la part de l'Université de Paris 13 (BQR 2016 ; chercheurs principaux: C. Matha, J.-F. Chiantaretto, I. Lasvergnas, V. Cornalba), et du Programme de recherche de l'IPA (2016; chercheure principale, I. Lasvergnas), ainsi que d'un support de l'UQÃM octroyé à I. Lasvergnas. Un second colloque relié au même projet de recherche sur le thème La consultation psychanalytique en institution: quels transferts? s'est tenu à Paris (Hôpital Sainte Anne) les 3 et 4 mars 2017. Les diverses communications présentées dans ce second colloque ont fait l'objet de publications dans les numéros 217, 218 et 219 (juin 2018, juillet 2018, septembreoctobre 2018) de la revue Carnet-Psy. Nous remercions ici l'ensemble des organismes subventionnaires qui ont permis la tenue de ces deux événements.

4. Pour mémoire, le CCTP Jean-Favreau, les BAPU (Bureaux d'aide psychologique universitaire) - dont le BAPU Pascal -, et le Centre Victor Smirnoff furent créés dans les années 1950. Le Centre Évelyne et Jean Kestemberg fut fondé au début des années 1970.

5. Ou autres "populations-cibles»: personnes pauvres ou au chômage, jeunes, étudiants, familles monoparentales, immigrants de fraîche date, personnes psychotiques, etc.

6. Une précision: contrairement à la situation des deux cliniques psychanalytiques québécoises existantes (la Clinique psychanalytique de Montréal - renommée en 2018 «Clinique sociale psychanalytique de Montréal» - et le GPMR) où les traitements offerts sont des traitements à bas tarifs et partiellement subventionnés, dans les cliniques françaises, les traitements sont gratuits parce que totalement pris en charge par les fonds publics - la question de la complète gratuité d'un traitement psychanalytique soulevant par ailleurs, du point de vue de l'inconscient, plusieurs questions importantes.

7. Ce dispositif original avait été le trouvé/créé collectif en 2007 de la part de l'équipe clinique fondatrice de la CPM. Il a été repris au GPMR, organisme fondé par des cliniciens qui avaient été partie prenante de «l'invention» de ce dispositif à la CPM (Lasvergnas, 2018 a ; voir également l'article dans le présent recueil).

8. On sait que l'attention portée aux mouvements transférentiels chez le patient projetés sur «le tiers institutionnel» ont permis à ces auteurs d'établir à titre de directeurs de ces organismes, des règles originales dans l'organisation de la prise en charge des patients: instauration du "personnage-tiers » au Centre Kestemberg et instauration d'un «double cadre» au CCTP Jean-Favreau, auxquelles on peut ajouter la contribution plus récente dans la mise en place d'un «double cadre» différent, et différemment théorisé, qui est celui du dispositif de la consultation à la CPM, et également adopté au GPMR.

9. De la référence paradigmatique de la «cure-type» divan/fauteuil plusieurs fois par semaine à un ensemble de dispositifs remaniés: rencontres en face-à-face à une fréquence moindre, thérapie par médiations (art-thérapie, musicothérapie, thérapie par médiation corporelle), ou encore psychodrame psychanalytique ou psychanalyse de groupe; sans omettre le profond remaniement du cadre de rencontre et de sa théorisation introduit par la technique de la scansion lacanienne. 


\section{Références}

Anzieu, D. (1976). La fantasmatique de la formation psychanalytique. Dans R. Kaës et al. (dir.), Fantasme et formation. Paris: Dunod, 2001.

Baldacci, J.-L. (2013). Fonctions de la consultation psychanalytique. Dans J. Bouhsira et M. Janin-Oudinot (dir.), La consultation psychanalytique (p. 11-28). Paris: Presses universitaires de France.

Donnet, J.-L. (2013). La rencontre consultative. Dans J. Boushira et M. Janin-Oudinot (dir.), La consultation psychanalytique (p. 29-50). Paris: Presses universitaires de France.

Donnet, J.-L. (2009). L'échange analytique: le récit et l'écoute. Dans auteur, L'humour et la honte. Paris: Presses universitaires de France.

Donnet, J.-L. (2005). La situation analysante. Paris: Presses universitaires de France.

Donnet, J.-L. (1997). Le site analytique et la situation analysante. Dans C. Couvreur, A. Oppenheimer, R. Perron, et J. Schaeffer (dir.), Psychanalyse, neurosciences, cognitivisme (p. 81-89). Paris: Presses universitaires de France.

Freud, S. (1926). La question de l'analyse profane. Paris: Gallimard, 2003.

Freud, S. (1919). Les voies nouvelles de la thérapeutique psychanalytique. Paris: Presses universitaires de France (1977).

Kaës, R. (2016). L’idéologie, l'idéal, l'idée, l'idole. Paris: Dunod.

Kaës, R. (2015). L'extension de la psychanalyse. Pour une métapsychologie de troisième type. Paris: Dunod.

Kaës, R. (2014). Les alliances inconscientes. Paris: Dunod.

Kaës, R. (2012). Le malêtre. Paris: Dunod.

Kapsambelis, V. (2012). Accueillir les patients psychotiques les plus gravement perturbés. Dans auteur, Quels toits pour soigner les personnes souffrant de troubles psychotiques? (p. 33-46). Toulouse: ERES.

Kapsambelis, V. (2017). Le Centre de psychanalyse de l'ASM13. Adolescence, 35 (1), 21-34.

Kestemberg, E. (1981). Le personnage tiers. Sa nature, sa fonction. Dans auteur (2001), La psychose froide (p. 144-147). Paris: Presses universitaires de France.

Lasvergnas, I. (2018 a). Naissance d'une clinique psychanalytique: entre héritages transférentiels et espace transitionnel partagé. Le Carnet-psy, 217, 44-49.

Lasvergnas, I. (2018 b). L'attraction du double dans la filiation: du socius à la transmission analytique. Psychanalyse et psychose, 18, 53-66. 